\title{
New Algorithms for Solving Tropical Linear Systems
}

\author{
Alex Davydow
}

August 19, 2021

\begin{abstract}
The problem of solving tropical linear systems, a natural problem of tropical mathematics, has already proven to be very interesting from the algorithmic point of view: it is known to be in $N P \cap \operatorname{coNP}$ but no polynomial time algorithm is known, although counterexamples for existing pseudopolynomial algorithms are (and have to be) very complex.

In this work, we continue the study of algorithms for solving tropical linear systems. First, we present a new reformulation of Grigoriev's algorithm that brings it closer to the algorithm of Akian, Gaubert, and Guterman; this lets us formulate a whole family of new algorithms, and we present algorithms from this family for which no known superpolynomial counterexamples work. Second, we present a family of algorithms for solving overdetermined tropical systems. We show that for weakly overdetermined systems, there are polynomial algorithms in this family. We also present a concrete algorithm from this family that can solve a tropical linear system defined by an $m \times n$ matrix with maximal element $M$ in time $\Theta\left(\left(\begin{array}{c}m \\ n\end{array}\right)\right.$ poly $\left.(m, n, \log M)\right)$, and this time matches the complexity of the best of previously known algorithms for feasibility testing.
\end{abstract}

\section{Introduction}

\subsection{Tropical mathematics and tropical linear algebra}

Tropical mathematics unites three closely connected fields of study: tropical algebra, tropical analysis, and tropical geometry. The term is usually taken to mean mathematics obtained from classical mathematics by replacing the addition and multiplication operations with minimum and addition respectively, hence the term min-plus algebra. Sometimes maximum is used instead of minimum, with perfectly symmetrical results, so in what follows we always use the minimum operation. Taking the minimum in tropical context is usually denoted by $\oplus$; addition, by $\otimes$. The $\oplus$ operation is idempotent, i.e., $a \oplus a=a$, so tropical mathematics is in fact a part of idempotent mathematics, although lately these notions have often been identified so that the term "tropical" is sometimes applied to any mathematical constructions with an idempotent operation. 
Tropical algebra was the first section of tropical mathematics to appear. The term originates from French mathematicians and first appears in the 1980s. Although different authors attribute the term to different researchers [31, 28, 21], all sources agree that the term came into general use in the honor of one of the founders of this field, a Brazilian mathematician Imre Simon, so the term "tropical" simply means how French mathematicians viewed Brazil. Simon himself uses the term already in the work [30] that laid out the foundations of tropical algebra, attributing the term to Christian Choffrut.

At first, the term "tropical" was used for the discrete version of $(\mathrm{min},+)$ algebra, but at present the terminology has shifted, and tropical algebra is usually meant to be an algebra over the semifield $\mathbb{R}_{\text {min }}$ (as stated above) or even, sometimes, an arbitrary algebra with an idempotent operation, e.g., (min, max) algebra. Both Simon and his French colleagues used tropical algebra for the study of finite state machines.

Although a systematic study of the tropical semiring began only after the works of Simon, we should note that the $(\min ,+)$ semiring had appeared before in optimization problems. For instance, Floyd's algorithm for finding shortest paths in a graph that was proposed in the 1960s [9] can be considered as taking a tropical degree of the distance matrix (tropical exponentiation is similar to classical with the difference that we replace addition with $\oplus$ and multiplication with $\otimes$ ). Speaking about idempotent algebra as a whole, the first work to make serious use of an algebra over an idempotent ring (apart from Boolean fields) was the work of Kleene [18] that studied nerve nets in the context of finite state machines. At present, there is a host of literature of matrices with idempotent coefficients and their applications, e.g., [5, 19, 22].

Tropical linear algebra is a subfield of tropical algebraic geometry that works with systems of tropical linear equations. Unfortunately, definitions remain a major problem in this subfield. Many notions of linear algebra have several equivalent definitions, but after tropicalization equivalence disappears, and we are left with several different definitions. One striking example of this phenomenon is that there may be several conflicting definitions for the root of a tropical polynomial.

In the case of the root of a polynomial, researchers finally settled on the definition of O. Viro, and now the set of roots of a polynomial are the set of this polynomial's nonsmoothness points. This definition prevailed because it preserves such important properties as, for instance, the fundamental theorem of algebra (that a polynomial of degree $n$ has exactly $n$ roots, counting multiplicities).

The problem of solving linear systems was formulated right after the definition of a root for a tropical polynomial was given, but the first work actually devoted to tropical linear algebra appeared only as late as 2005 [7. At present, this field is primarily being developed in France (Akian, Gaubert, Grigoriev and others), sometimes in collaboration with researchers from other countries (Izhakian, Guterman).

Since there are no known efficient algorithms for the main problems of tropical linear algebra, it is currently little used in practice. Nevertheless, there are practical problems that would benefit from developments in this field. For instance, Noel, Grigoriev, Vakulenko, and Radulescu have recently proposed a way to use algorithms for solving tropical linear systems 
to study stable states of reaction networks in biology [27, 26]. Thus, problems of tropical linear algebra are important from both theoretical and practical points of view.

It is worth noting that many definitions and theorems of tropical geometry (including, in particular, tropical linear algebra) appeared much earlier than tropical geometry itself took shape as a field of study. In these cases, tropical geometry serves as a language in which it is convenient to state theorems that have already been proven from a different viewpoint. Although at first glance this serves little useful purpose, sometimes such a translation may lead to new results. For instance, a translation of the Viro's patchworking method to the language of tropical geometry has led Mikhalkin to an algorithm for computing GromovWitten invariants [25].

\subsection{Tropical linear systems}

After researchers had agreed on the definitions of a tropical polynomial and tropical root, one of the first problems that they tried to solve was constructing an algorithm for testing the feasibility of a linear tropical system. However, unlike the classical case the tropical problem turned out to be much harder, and despite the fact that it was put forward five years ago, no efficient solution is known to date.

Similar to the classical case, a tropical linear system can be conveniently defined with a matrix. One way to test the feasibility of a classical linear system is to test whether its determinant is zero. Therefore, due to the idempotent correspondence principle, one could expect something similar in the tropical case as well. A formula for the tropical determinant was first proposed by Izhakian in 2008 [13]. The determinant was defined completely similar to the classical determinant with the sole difference that one uses $\oplus$ instead of addition, $\otimes$ instead of multiplication, and there is no $(-1)^{n}$ factor (i.e., this construction corresponds to the determinant and the permanent at the same time). Izhakian also showed that a tropical system defined by a square matrix has a solution if and only if its coefficients are not roots of the tropical determinant (i.e., the determinant of this matrix is minimized only on a single monomial). Such square matrices were called tropical singular. Interestingly, 14 years before Izhakian, Butcovič already defined tropical singularity under the name of strong regularity [4]. However, it was done before tropical geometry appeared in earnest, and Butcovič did not establish any connection between strong regularity and feasibility of tropical systems.

It is important to note that Butcovič proposed an efficient algorithm for testing a tropical matrix for singularity 4. One can also note that the singularity condition is equivalent to the existence of a unique minimal weight matching and can therefore be efficiently tested with, e.g., the Hungarian method [20]. Thus, the feasibility problem for tropical linear systems defined by square matrices was efficiently solved by Izhakian in 2008. However, for other matrices even pseudopolynomial algorithms (that would work in time polynomial of the numerical value of the input rather than its size) were not known.

In 2009, Izhakian generalized his definition to rectangular matrices [14] and showed that a system defined by a rectangular matrix is infeasible if and only if it contains a singular submatrix of maximal width (i.e., width equal to the width of the matrix). Note that Izhakian's results imply that similar to the classical case, a tropical system with fewer equations than 
variables is always feasible. However, since a rectangular matrix may contain an exponential number of square submatrices of maximal width (in case when the height is much larger than the width), this result did not imply an efficient algorithm for solving tropical linear systems defined by rectangular matrices. We should also note that in the case of finite coefficients Izhakian's results are in fact a simple corollary of the theorem that establishes that Kapranov rank and tropical rank of a matrix are maximized simultaneously, a theorem proven by Develin, Santos, and Sturmfels in 2005 [7].

In case when the number of equations and variables coincide, one can not only efficiently test the system for feasibility but also solve it. To do so, Grigoriev proposed[10] to drop the equation that intersects with minimal matchings in two cells and apply the tropical Cramer's rule to the rest of the matrix [29]. Thus, the problem of solving a tropical system with a square matrix can be solved in polynomial time.

The first pseudopolynomial algorithm for solving rectangular matrices was presented by Akian, Gaubert, and Guterman in 2010 [1]. They showed that the feasibility problem for a tropical linear system has a polynomial reduction to the problem of finding the winner in mean payoff games. Mean payoff games were proposed by Ehrenfeucht and Mycielski in 1979 [8] and have already been quite comprehensively studied by 2010. For instance, in 1993 Karzanov and Lebedev, using the results of Karp [15], showed that the problem of finding the winner in a mean payoff game lies in the intersection of complexity classes $N P$ and coNP [17]; in 1995, Zwick and Paterson proposed a pseudopolynomial algorithm for solving this problem [32]. Several times, there appeared algorithms that claimed to find the winner of a mean payoff game in polynomial time [16, 23, 24], but so far all of them turned out to contain mistakes [32].

Thus, it was shown in 2010 that the feasibility problem for a tropical system lies in the intersection of $N P$ and $c o N P$; this is an interesting complexity class: there are few problems known to be in $N P \cap c o N P$ but not known to be in $P$, and most of them are polynomially equivalent to each other. The feasibility problem also got a pseudopolynomial algorithm. However, it is easy to construct an example of a matrix that results, by the algorithm of Akian, Gaubert, and Guterman, in a mean payoff game with no known efficient algorithm. Therefore, the problem of finding an efficient algorithm for testing feasibility of a tropical linear system remained open.

In 2011, Grigoriev proposed a different pseudopolynomial algorithm [11] similar to the Gram-Schmidt process that is designed to immediately solve the feasibility problem for a tropical system. Apart from its relative simplicity, an important feature of Grigoriev's algorithm was that apart from the pseudopolynomial estimate he immediately found an exponential complexity bound that does not depend on the numbers that occur in the matrix. Later Davydow showed [6] that if one fixes any one of the three parameters (width, height, and maximal coefficient in the matrix), the algorithm will work in time polynomial with respect to the other two parameters. These were optimistic results: they meant that if there were examples of inputs on which Grigoriev's algorithm is not polynomial, they would have to be very complex since all three parameters would have to change simultaneously. Unfortunately, in 2012 Davydow found such a series [6], and by the time Grigoriev's work 
was published it was already known that this algorithm is also not efficient in the general case.

As we have already mentioned, in 2010 Akian et al. [1] showed that the feasibility problem for a tropical linear system can be polynomially reduced to the problem of finding the winner in mean payoff games. In 2012, Grigoriev and Podolskii [12 found the inverse reduction, showing that the feasibility problem for a tropical linear system and the winner problem in a mean payoff game are equivalent. They constructed a reduction to the problem of $(\mathrm{min},+)$ systems (systems of equations of the form $A x=B x$, where matrices are multiplied by vectors in the tropical sense, and the equivalence of the problem of $(\mathrm{min},+)$ systems to the problem of mean payoff games was proven by Bezem, Nieuwenhuis, and Rodrígez-Carbonell already in 2010 [3]. At the same time, a similar result was directly obtained by Akian, Gaubert, and Guterman 2].

On one hand, after Grigoriev and Podolskii showed that testing feasibility for a tropical system is as hard as finding the winner in a mean payoff game, it became clear that finding an efficient algorithm is rather unlikely. On the other hand, they showed that this problem is interesting not only as an independent problem but also as a completely new approach to mean payoff games, and that this problem deserves an even more detailed scrutiny.

Thus, at present there exist efficient feasibility testing algorithms for systems where the number of equations exceeds the number of variables by a predefined constant; we will call such systems weakly overdetermined. In this case, it suffices to enumerate all square submatrices of maximal width for the system's matrix and test each of them for singularity with the Hungarian method. Then, Izhakian's results imply that a system if feasible if and only if all resulting matrices are nonsingular. For systems with an unbounded number of equations, only pseudopolynomial algorithms are known (algorithm of Akian-GaubertGuterman and Grigoriev's algorithm). Note that in case of systems with bounded number of variables Grigoriev's algorithm works in polynomial time.

\subsection{Our contributions}

In this work, we present two main results related to new algorithms for solving tropical linear systems. First, we present a new description for Grigoriev's algorithm for solving tropical linear systems that lets us generalize Grigoriev's algorithm and another well known algorithm of Akian, Gaubert, and Guterman and consider a whole family of algorithms that differ in the lifting operation. Even a simple straightforward combination of these two algorithms can already solve hard counterexamples for both Grigoriev's and Akian-Gaubert-Guterman algorithms, and we leave devising hard counterexamples for these new algorithms as an interesting open problem.

Second, we present a new algorithm for overdetermined tropical linear systems, i.e., systems that have more equations than variables. We reduce solving an overdetermined tropical system to solving several of its subsystems. This leads to a general algorithm that works on all tropical systems and runs in time $\Theta\left(\left(\begin{array}{c}m \\ n\end{array}\right)\right.$ poly $\left.(m, n, \log M)\right)$. Moreover, we show that weakly overdetermined tropical systems (where equations outnumber variables only by a predefined constant) admit a polynomial time solution. 
The paper is organized as follows. In Section 2, we give a formal definition of the feasibility problem for a tropical system and introduce the notation used throughout the paper. Section 3 is devoted to optimizing Grigoriev's algorithm for solving linear tropical systems; our optimization leads to a unified approach for Grigoriev's algorithm together with the algorithm of Akian, Gaubert, and Guterman, which in turn lets us combine the two algorithms, getting an algorithm with no known counterexamples where it would have to work for superpolynomial time. Finally, as we have already mentioned, it is possible to test feasibility of weakly overdetermined tropical linear systems in polynomial time. Section 4 presents a novel algorithm that actually solves such systems in polynomial time. Section 5 concludes the paper.

\section{Problem setting}

In this section, we give basic definitions regarding tropical linear systems.

Definition 1. A tropical linear system is a rectangular matrix of size $m \times n$. A solution of a tropical linear system is a row of $n$ elements such that after adding it to each row of the matrix each sum does not contain a strict minimum, i.e., the minimal element occurs at least twice in every row. A tropical linear system is called feasible if there exists a row that is a solution of this system [11].

Example 1. For the matrix

$$
\left(\begin{array}{lll}
1 & 2 & 3 \\
3 & 2 & 1
\end{array}\right),
$$

the row

$$
\left(\begin{array}{lll}
1 & 0 & 1
\end{array}\right)
$$

represents a solution: after adding it to the first row we get the minimal value 2 in the first and second columns; for the second row, we get the minimal value 2 in the second and third columns.

Example 2. The matrix

$$
\left(\begin{array}{ll}
1 & 2 \\
3 & 2
\end{array}\right)
$$

is obviously infeasible.

In this work we will consider the problem of establishing feasibility for integer-valued tropical linear systems. First, we note that we can apply some simple transformations to a system's matrix without changing its feasibility status. The following proposition is obvious.

Proposition 1. The class of feasible tropical linear systems is invariant with respect to adding an arbitrary constant to all numbers in one row or in one column. Moreover, given a solution of the system after such a transformation, one can find a solution of the original system by adding to the solution the difference between first rows of the matrix before and after the transformation. 
Proposition 1 immediately implies the following remark.

Remark 1. Without loss of generality we can assume that all elements of the system's matrix are nonnegative.

In what follows we introduce the following notation for a matrix $A$ :

- $m(A)$, the number of rows in the matrix $A$,

- $n(A)$, the number of columns in the matrix $A$,

- $k(A)=n(A)-m(A)$,

- $M(A)$, the maximal number in the matrix $A$,

- $R(A)$, the set of rows of the matrix $A$.

- $a_{i}(A)$, the $i^{\text {th }}$ row of the matrix $A$.

We will omit the argument in this notation if it is clear from context what matrix we are talking about.

\section{Grigoriev's algorithm and its modifications}

\subsection{The original algorithm}

One recently proposed algorithm for solving tropical linear systems is Grigoriev's algorithm. As we have already noted, a key feature of this algorithm is that the work [1] that proposes this algorithm immediately shows both an upper bound on the algorithm's complexity that polynomially depends on the matrix size (but it is polynomial in $M$, the largest element of the matrix, rather than $\log M$ ) and an upper bound that polynomially depends on $\log M$ (but it is not polynomial in matrix size).

We begin with a description of Grigoriev's algorithm. We begin by noting that due to Proposition 1 we can find not a solution of the matrix but rather a series of transformations that consists of adding a constant to all elements in a row or in a column that would reduce the original matrix to a matrix that has a zero row for a solution. In what follows, we call such a matrix the solution matrix; finding it is equivalent to finding a solution.

To solve a system of size $m \times n$, we proceed by induction and assume that we have solved the system of size $(m-1) \times n$ obtained from the initial system by removing its first row. From this moment on we will assume that all rows of the matrix, except possibly the first row, do not contain strict minima. Next we define the lifting operation with Algorithm 1 .

Note that although the lifting algorithm does contain some indeterminacy: it is not specified in what order we add columns to the set $J$, when the first loop ends the set $J$ is uniquely defined since the maximal by inclusion such set is unique.

Then we transform the matrix according to Algorithm 2, The time complexity of this algorithm was originally shown to be $O\left(m^{2} n^{2} M \log M\right)$ [11. Later, a different estimate of 

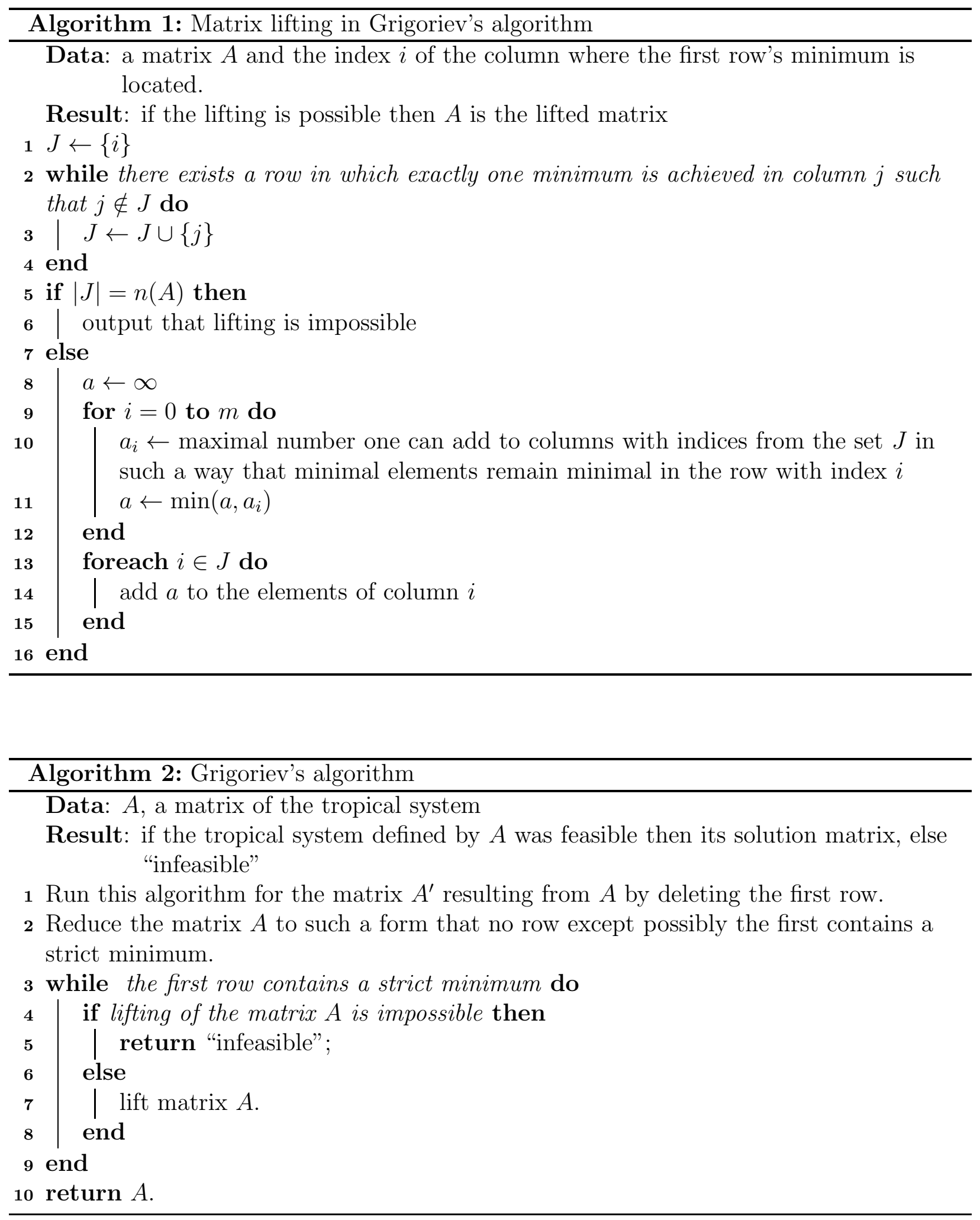
$O\left(\log M \cdot m \cdot n^{2} \cdot\left(\begin{array}{c}m+n \\ n\end{array}\right)\right)$ was shown by Davydow [6]. Besides, there is a known counterexample for Grigoriev's algorithm: there exists a sequence of matrices (with unbounded growth in the number of rows and columns) such that Grigoriev's algorithm takes $\Omega\left(n^{\frac{m}{6}} \log M\right)$ time to process them, where $M=\operatorname{poly}\left(n^{\frac{m}{6}}\right)[6]$.

\subsection{Properties of the solutions found by Grigoriev's algorithm}

We introduce a partial ordering on the solutions of a tropical linear system: we say that one solution is less than the other if it is smaller componentwise. Then the following theorem holds.

Theorem 1. For a matrix with one strict minimum, Grigoriev's algorithm finds the smallest nonnegative solution. In terms of solution matrices, Grigoriev's algorithm finds the smallest solution matrix which is greater than the original matrix.

Proof. First note that the existence of such a solution follows from the well-known fact that the set of solutions is linear.

We prove this theorem by induction on the number of liftings. Namely, we show that on each step of Grigoriev's algorithm the matrix does not become less than the maximal solution matrix among those that are smaller than the original matrix. For the induction base, note that the original matrix obviously satisfies this condition.

For the induction step, note that if, during a lifting, we add to at least one column a number smaller than the one added in the algorithm, then the column to which we added the smallest number will have a strict minimum. This means precisely that in the smallest solution matrix among those that larger than the original matrix we have to add at least as much as Grigoriev's algorithm adds.

\subsection{Optimizing Grigoriev's algorithm}

We begin with a simple corollary of Theorem 1 .

Corollary 1. If Grigoriev's algorithm has changed every column at least once, it will output "infeasible".

Proof. In the smallest solution, at least one of the elements must be zero; otherwise, one could subtract it from every element and get a smaller solution.

Corollary 1 implies our first optimization of Grigoriev's algorithm: we can interrupt it and output "infeasible" not when lifting is impossible, but rather when each column has been changed at least once, which can happen much earlier.

For a second optimization, we can also do without the recursion on the matrix height: we can simply add all columns with a strict minimum to the set $J$ from the very beginning. Note that while the first optimization obviously cannot hurt Grigoriev's algorithm, we do not know this for the second idea, although we have failed to find an example where the original version of the algorithm would work faster than this modification. 
For this version of Grigoriev's algorithm, the same upper and lower bounds can be proven in the same way as for the original version. Apart from some simplification, this version of Grigoriev's algorithm has the advantage that it is now very similar to the AkianGaubert-Guterman algorithm; the only difference remains in the lifting operation: the Akian-Gaubert-Guterman algorithm lifts only columns with strict minima, and only for the value needed in order for the minima to cease being strict (see Algorithm 4).

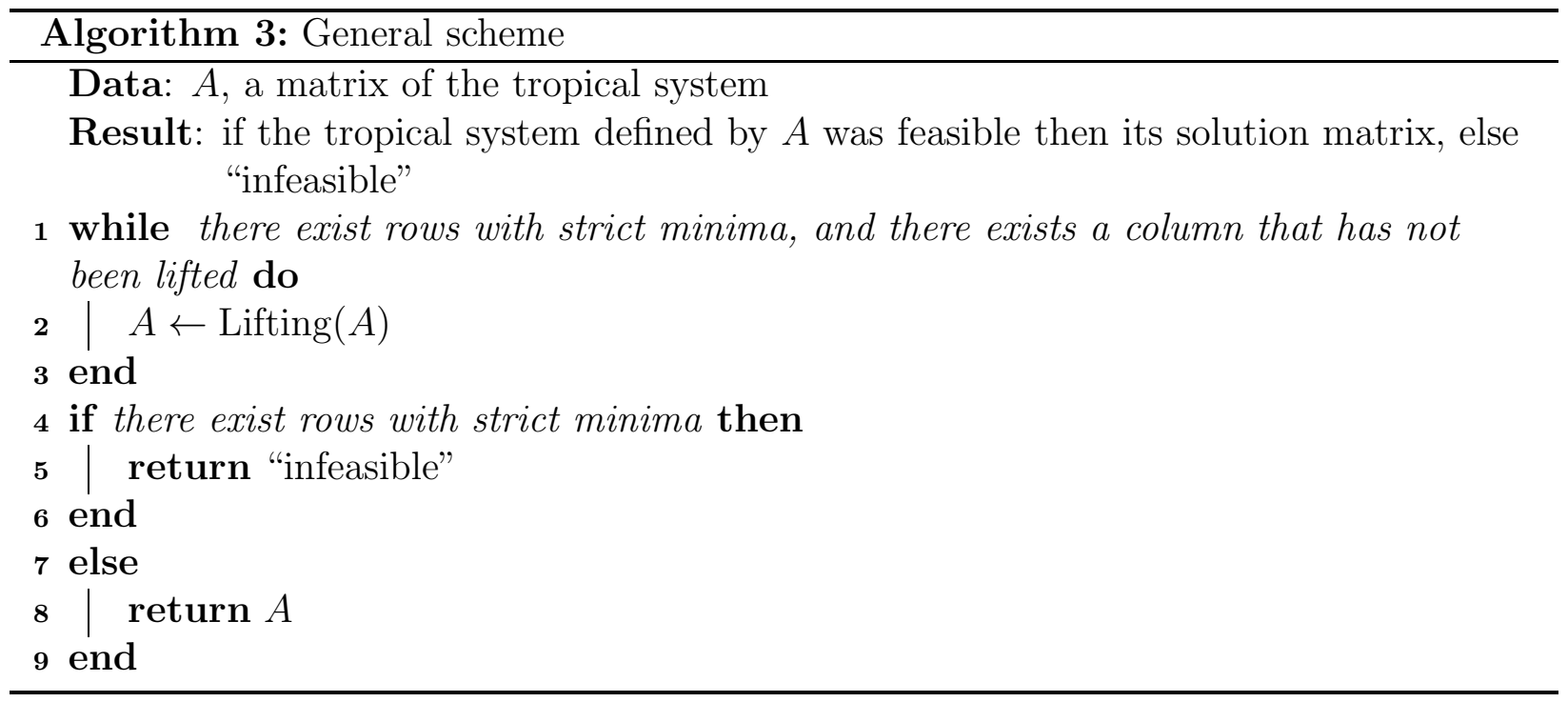

This leads us to considering an entire scheme of algorithms that differ only in the lifting operation; their general scheme is shown in Algorithm 3. All we need from this operation is that after the lifting the matrix does not exceed the minimal solution that is larger than the matrix before the lifting. For instance, on the lifting step we can add to each column the maximum of the numbers that Akian-Gaubert-Guterman algorithm and Grigoriev's algorithm propose to add to this column. It is easy to construct even better lifting methods, but at present, we do not know a superpolynomial counterexample even to this simple combination of the Akian-Gaubert-Guterman algorithm and Grigoriev's algorithm, shown in Algorithm 6. Finding such a counterexample remains an interesting open problem that could shed light on important properties of this class of algorithms.

\section{$4 \quad$ Weakly overdetermined systems}

In this section, we proceed to the second main result of this work, namely an algorithm for solving overdetermined tropical systems. In the following theorem, we show how to reduce solving an overdetermined tropical system of width $n$ to solving $n+1$ systems corresponding to its submatrices. Throughout the section, we will assume that arithmetic operations with numbers in the matrix take $O(1)$ time. 

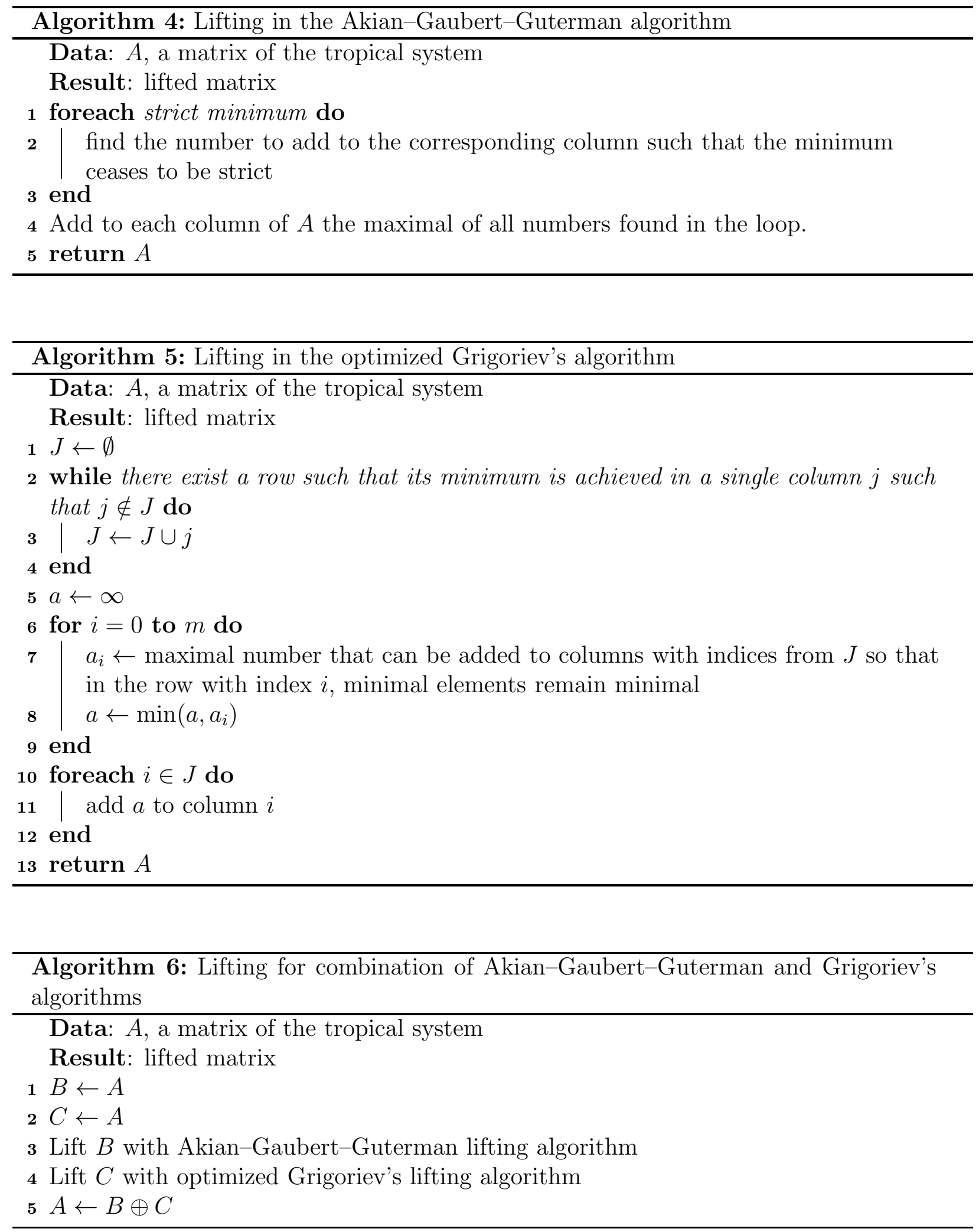
Theorem 2. Consider a matrix $A$ and $n(A)+1$ subsets of its set of rows such that every row of $A$ is covered at least $n$ times by these subsets. Then, if each subset defines a feasible tropical system, $A$ is also feasible. Moreover, if solutions of each of these systems are known, the solution of matrix $A$ can be found in polynomial time.

Proof. We begin by constructing a matrix of solutions $S$ with rows $s_{i}$, where $s_{i}$ is the solution for the system defined by the $i^{\text {th }}$ subset. Then we find a solution for the tropical system $S^{\top}(S$ transposed), denoting it by $\alpha$; this solution exists and can be found in polynomial time since $S^{\top}$ is underdetermined: the number of equations is less than the number of variables. Note that $s_{i}+\alpha_{i}$ is still a solution for the system defined by the $i^{\text {th }}$ subset since multiplication by a tropical constant preserves a solution. Consider $x=\bigoplus_{i}\left(s_{i}+\alpha_{i}\right)$. Then $x$ is the solution for the original matrix $A$.

Indeed, since $\alpha$ is a solution, we can remove any row from this tropical sum, and $x$ will remain unchanged (because every minimum is achieved twice). Since each of the rows $a_{i}$ is covered at least $n$ times, by dropping the row corresponding to a set that does not cover $a_{i}$ we get a solution for $a_{i}$ since the set of solutions is linear.

Example 3. Let us consider how this algorithm works on the following example with an overdetermined matrix

$$
\left(\begin{array}{lll}
1 & 2 & 3 \\
1 & 2 & 1 \\
1 & 2 & 5 \\
2 & 3 & 1
\end{array}\right)
$$

We begin by choosing 4 subsets of its rows:

$$
\left(\begin{array}{lll}
1 & 2 & 3 \\
1 & 2 & 1 \\
1 & 2 & 5
\end{array}\right),\left(\begin{array}{lll}
1 & 2 & 3 \\
1 & 2 & 1 \\
2 & 3 & 1
\end{array}\right),\left(\begin{array}{lll}
1 & 2 & 3 \\
1 & 2 & 5 \\
2 & 3 & 1
\end{array}\right),\left(\begin{array}{lll}
1 & 2 & 1 \\
1 & 2 & 5 \\
2 & 3 & 1
\end{array}\right) .
$$

First we construct the matrix of solutions $S$ and its transpose:

$$
S=\left(\begin{array}{lll}
1 & 0 & 0 \\
2 & 1 & 2 \\
3 & 2 & 3 \\
2 & 1 & 2
\end{array}\right), \quad S^{\top}=\left(\begin{array}{cccc}
1 & 2 & 3 & 2 \\
0 & 1 & 2 & 1 \\
0 & 2 & 3 & 2
\end{array}\right)
$$

Next we solve $S^{\top}$, getting the solution matrix

$$
\left(\begin{array}{llll}
3 & 2 & 3 & 2 \\
2 & 1 & 2 & 1 \\
2 & 2 & 3 & 2
\end{array}\right) \text { and its transpose }\left(\begin{array}{lll}
3 & 2 & 2 \\
2 & 1 & 2 \\
3 & 2 & 3 \\
2 & 1 & 2
\end{array}\right)
$$

and find the tropical sum of its rows:

$$
x=\left(\begin{array}{lll}
2 & 1 & 2
\end{array}\right) .
$$

The resulting $x$ is a solution for the original matrix. 
Remark 2. As it often happens in tropical mathematics, this theorem holds in the classical case as well. Indeed, the $n+1$ vectors that represent solutions for subsets of equations are necessarily linearly dependent. This means that there exists a vector $x$ that can be expressed as a linear combination of the other vectors; by the reasoning similar to the proof of Theorem 2, this vector will be a solution for the original problem.

The most straightforward way to turn Theorem 2 into an algorithm is to choose on each step $n+1$ subsets with $n+k-1$ rows each, making sure that for each of the sets the absent rows are different. Thus, the feasibility problem for the original matrix can be reduced to $n+1$ problems of smaller size. To estimate the complexity of the resulting algorithm in terms of $n$ and $k$, we denote this complexity by $T(n, k)$. Recall that $T(n, 0)=\operatorname{poly}(n)$. We get the following recurrent relation for $T(n, k): T(n, k)=(n+1) T(n, k-1)+\operatorname{poly}(n)$. This means that $T(n, k)=(n+1)^{k}$ poly $(n)$, which is a polynomial for $k$ bounded by a constant, so we have arrived at the following theorem.

Theorem 3. The problem of solving weakly overdetermined tropical linear systems (systems for which $k$ is bounded by a constant) can be solved in polynomial time.

One can consider other ways of choosing the subsets. One of the most efficient methods is the following: consider a matrix $A$ for which we need to find a solution. First, we introduce an ordering on the rows of a matrix corresponding to the order of rows in the matrix $A$; we will further assume that rows in all subsets are ordered in this way. On each step, we choose $n+1$ subsets as follows: the first $n$ rows, all rows except the first, all rows except the second, and so on, ending with all rows except the $n^{\text {th }}$. To further improve the algorithm's running time, we use dynamical programming, storing the matrices that appear over the course of the algorithm's operation and their corresponding solutions in order to reuse them if the same matrix appears for a second time.

After this optimization, all we need to estimate the running time is to estimate the number of submatrices appearing in the algorithm. Note that all submatrices look as follows: the first $n$ rows are an arbitrary ordered subset of rows, and the rest are always several consecutive last rows. Thus, the number of matrices can be bounded from above by $m\left(\begin{array}{c}m \\ n\end{array}\right)$ (there are $\left(\begin{array}{c}m \\ n\end{array}\right)$ ways to choose the first $n$ rows, and the other rows are uniquely defined by their number). As a result, we get that the algorithm for solving weakly overdetermined systems has complexity $\left(\begin{array}{l}m \\ n\end{array}\right)$ poly $(m, n)$, which up to a polynomial coincides with the upper bound for the best known algorithm for feasibility testing in tropical linear systems. This bound is better than the upper bound on Grigoriev's algorithm but worse than the best known lower bound. With this algorithm, outlined in Algorithm 7, we have finally proven our main result in this section.

Theorem 4. Any tropical linear system can be solved in time

$$
\Theta\left(\left(\begin{array}{c}
m \\
n
\end{array}\right) \operatorname{poly}(m, n, \log M)\right) .
$$




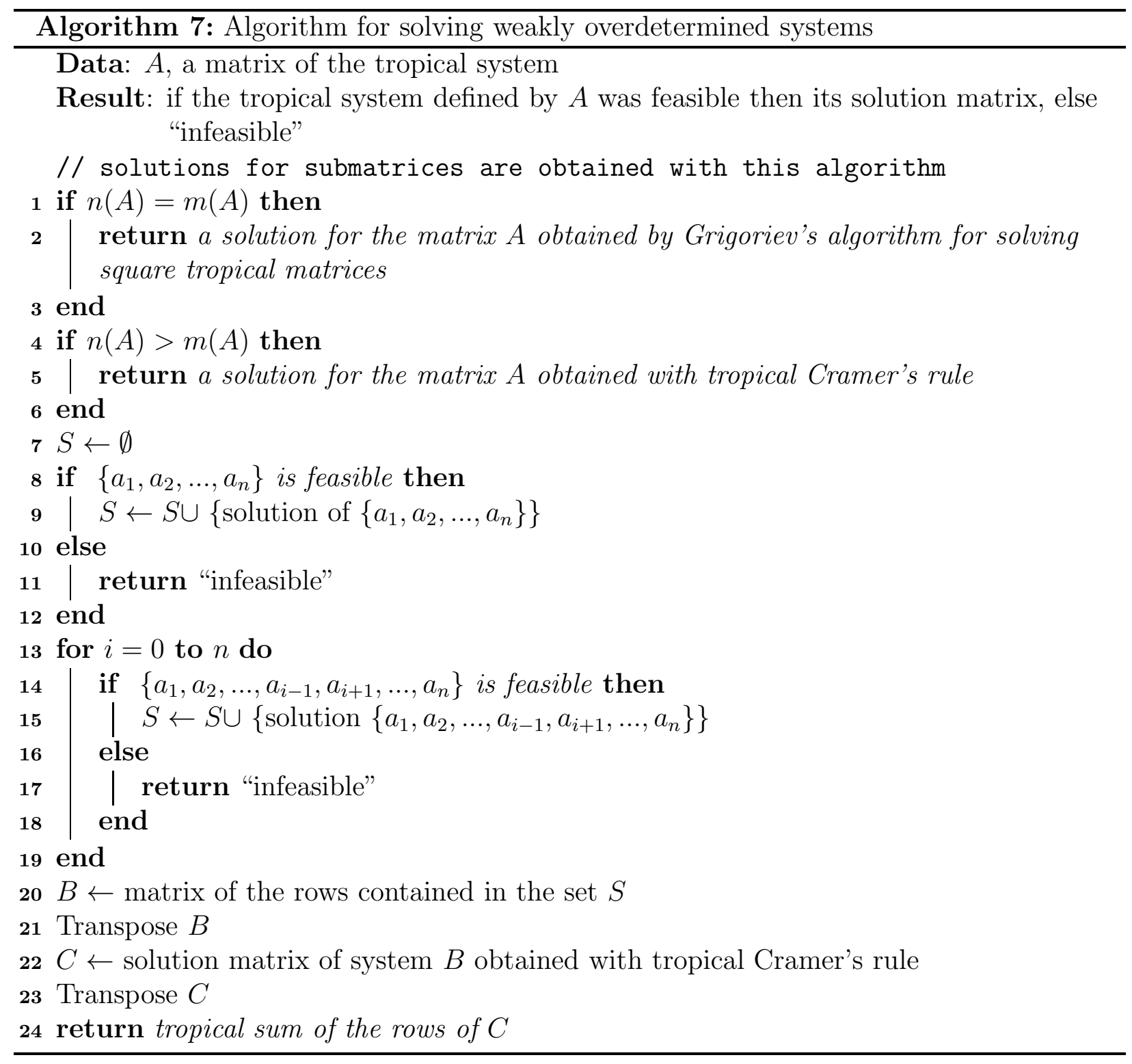




\section{Conclusion}

In this work, we have presented new algorithms for solving tropical linear systems: a modification of Grigoriev's algorithm that leads to a new family of algorithms with different lifting operations and a novel algorithm for solving overdetermined tropical systems that has the same time complexity as the previously known feasibility testing algorithm.

Tropical linear systems turn out to have very interesting computational properties: the problem lies in $N P \cap c o N P$ but no polynomial algorithm is known, and the best algorithm known so far was polynomial in any two of its characteristics out of three (width, height, and maximal element in the system's matrix). Further work in this direction may include further improvements of the algorithms proposed in this paper and finding counterexamples for the new algorithms proposed here: while we do claim that we have made Grigoriev's algorithm significantly faster in practice, we doubt that a simple combination of Grigoriev's algorithm and the Akian-Gaubert-Guterman is indeed polynomial, so we expect the search for hard counterexamples to succeed.

Another interesting direction for further study comes from the notion that for every linear set of points there is a minimal tropical prevariety (with respect to inclusion) that contains this set. In low dimensions such a prevariety can be constructed as a closure of the original set under several simple operations, and one can test infeasibility by testing if a prevariety built on a set of vectors contains the entire space. We believe that this approach may lead to new algorithms for solving tropical linear systems.

\section{References}

[1] M. Akian, S. Gaubert, and A. Guterman. The correspondence between tropical convexity and mean payoff games. Proc. 19 Intern. Symp. Math. Theory of Networks and Systems, pages 1295-1302, 2010.

[2] M. Akian, S. Gaubert, and A. Guterman. Tropical polyhedra are equivalent to mean payoff games. International Journal of Algebra and Computation, 22(1), 2012.

[3] M. Bezem, R. Nieuwenhuis, and Rodrígez-Carbonell E. Hard problems in max-algebra, control theory, hypergraphs and other areas. Inf. Process. Lett., 110(4):133-138, 2010.

[4] P. Butcovič. Strong regularity of matrices - a survey of results. Discrete Applied Mathematics, 48:45-68, 1994.

[5] R. A. Cuninghame-Green. Minimax algebra and applications. Springer Lect. Notes in Economics and Mathematical Systems, 166, 1979.

[6] A. P. Davydow. Upper and lower bounds for grigoriev's algorithm for solving integral tropical linear systems. Journal of Mathematical Sciences, 192(3):295-302, 2013.

[7] M. Develin, F. Santos, and Sturmfels B. On the rank of a tropical matrix. Math. Sci. Res. Inst. Publ., Combinatorial and computational geometry, 52:213-242, 2005. 
[8] A. Ehrenfeucht and J. Mycielski. Positional strategies for mean payoff games. International Journal of Game Theory, 8:109-113, 1979.

[9] Robert W. Floyd. Algorithm 97: Shortest path. Commun. ACM, 5(6):345-, June 1962.

[10] D. Grigoriev. Personal communication.

[11] D. Grigoriev. Complexity of solving tropical linear systems. Comput. Complexity, 22:71-78, 2013.

[12] D. Grigoriev and V. V. Podoltkii. Complexity of tropical and min-plus linear prevarieties. CoRR, abs/1204.4578, 2012.

[13] Z. Izhakian. The tropical rank of a tropical matrix. Eprint arXiv:math.AC/0604208v2, 2008.

[14] Z. Izhakian and L. Rowen. The tropical rank of a tropical matrix. Communications in Algebra, 37(11):3912-3927, 2009.

[15] R. M. Karp. A characterization of the minimum cycle mean in a digraph. Discrete Mathematics, 23:309-311, 1978.

[16] A. V. Karzanov, V. A. Gurvich, and L. G. Khaciyan. Cyclic games and an algorithm to finnd minimax cycle means in directed graphs. USSR Computational Mathematics and Mathematical Physics, 28:85-91, 1988.

[17] A. V. Karzanov and V. N. Lebedev. Cyclical games with prohibitions. Mathematical Programming, 60:277-293, 1993.

[18] S. C. Kleene. Representation of events in nerve nets and finite automata. Automata Studies, ed. by C.E. Shannon and J. McCarthy. Annals of Mathematics Studies, 34, 1956.

[19] N. K. Krivulin. Methods of idempotent algebra in problems of complex systems modeling and analysis. St. Petersburg Univ. Press, 2009.

[20] Harold W. Kuhn. The hungarian method for the assignment problem. Naval Research Logistics Quarterly, 2:83-97, 1955.

[21] G. L. Litvinov. The Maslov dequantization, idempotent and tropical mathematics: A brief introduction. Journal of Mathematical Science, 140(3), July 2007.

[22] G. L. Litvinov, V. P. Maslov, Rodionov A. Ya., and A. N. Sobolevski. Universal algorithms, mathematics of semirings and parallel computations. Lecture Notes in Computational Science and Engineering, 75, 2011.

[23] D. D. Lozovanu. Algorithms to solve some classes of network minimax problems and their applications. Cybernetics, 29:93-100, 1991. 
[24] D. D. Lozovanu. Strongly polynomial algorithms for nding minimax paths in networks and solution of cyclic games. Cybernetics and Systems Analysis, 29:754-759, 1993.

[25] G. Mikhalkin. Enumerative tropical algebraic geometry in R2. ArXiv Mathematics e-prints, December 2003.

[26] V. Noel, D. Grigoriev, S. Vakulenko, and O. Radulescu. Hybrid models of the cell cycle molecular machinery. Electronic Proceedings in Theoretical Computer Science, 92:88-105, 2012.

[27] V. Noel, D. Grigoriev, S. Vakulenko, and O. Radulescu. Tropical geometries and dynamics of biochemical networks. application to hybrid cell cycle models. $S A S B$ 2011, Electronic Notes in Theoretical Computer Science, 284:75-91, 2012.

[28] Jean-Eric Pin. Positive varieties and infinite words. In LATIN, pages 76-87, 1998.

[29] J. Richter-Gebert, B. Sturmfels, and T. Theobald. First steps in tropical geometry. ArXiv Mathematics e-prints, June 2003.

[30] Imre Simon. Recognizable sets with multiplicities in the tropical semiring. In MichalP. Chytil, Václav Koubek, and Ladislav Janiga, editors, Mathematical Foundations of Computer Science 1988, volume 324 of Lecture Notes in Computer Science, pages 107120. Springer Berlin Heidelberg, 1988.

[31] D. Speyer and B. Sturmfels. Tropical Mathematics. ArXiv Mathematics e-prints, August 2004.

[32] U. Zwick and M. Paterson. The complexity of mean payoff games on graphs. Theoret. Comput. Sci., 158(1-2):342-359, 1995. 